\title{
Dietary factors that influence maternal iodine status in UK pregnant women
}

\author{
S. C. Bath ${ }^{1}$, C. D. Steer ${ }^{2}$, P. M. Emmett ${ }^{2}$, J. Golding ${ }^{2}$ and M. P. Rayman ${ }^{1}$ \\ ${ }^{1}$ University of Surrey, Guildford, Surrey, GU2 $7 X^{2}{ }^{2}$ University of Bristol, Bristol, BS8 1TH, UK
}

An adequate intake of iodine is vital during pregnancy as iodine is required for fetal brain development ${ }^{(1)}$. We have previously shown that a low iodine status in UK pregnant women is adversely associated with child cognition (IQ and reading ability) up to the age of nine years ${ }^{(2)}$. As the UK is now classified as mildly iodine deficient and has no national policy for ensuring adequate iodine status, such as salt iodisation, it is important to understand the dietary factors that contribute to iodine status in pregnancy.

Our study investigated maternal iodine status in women of the Avon Longitudinal Study of Parents and Children (ALSPAC) cohort who were recruited in the $1990 \mathrm{~s}^{(3)}$. We measured iodine concentration (and creatinine to adjust for urine volume) in urine samples collected from 1475 women throughout pregnancy [median gestational age 11 (IQR 9-13) weeks]. Women completed a Food Frequency Questionnaire (FFQ) during pregnancy ${ }^{(4)}$ and, for the purposes of comparing to urinary iodine excretion, six iodine-rich food items were selected [i.e. milk, cheese, eggs (and quiche), seafood (white, oily and shellfish) meat/poultry, and grains (rice, pasta and bread)]. The FFQ answers were converted to weekly number of portions for single items (eggs and cheese) or weekly intake (grams) ${ }^{(4)}$ for composite food groups (seafood, milk, meat/poultry and grains) and then recoded to tertiles.

The group was classified as iodine-deficient ${ }^{(1)}$, with a median urinary iodine concentration of $92.6 \mu \mathrm{g} / \mathrm{L}$ (iodine/creatinine ratio $114 \mu \mathrm{g} / \mathrm{g})$. The iodine/creatinine ratio significantly differed between tertiles of weekly milk $(p<0.001)$ and seafood $(p=0.03)$ consumption and between the number portions of egg consumed per week $(p=0.01)$. After adjustment for all six food categories, maternal age and gestational week of urine sample, a low iodine status $(<150 \mu \mathrm{g} / \mathrm{g})$ was significantly associated with low milk and egg consumption (Table); maternal age was independently positively associated with the iodine/creatinine ratio $(p=0.001)$.

\begin{tabular}{lllc}
\hline & & \multicolumn{2}{c}{ Risk of urinary iodine status $<150 \mu \mathrm{g} / \mathrm{g} *$} \\
\cline { 3 - 4 } Food Group & Weekly consumption level & \multicolumn{1}{c}{ OR $(95 \% \mathrm{CI})$} & $P$ value \\
\hline Milk & Bottom tertile & 1 & 0.003 \\
& Middle tertile & $0.62(0.47,0.82)$ & \\
\multirow{3}{*}{ Eggs } & Top tertile & $0.71(0.54,0.94)$ & 0.02 \\
& $<$ Half portion & 1 & \\
& $1-3$ portions & $0.90(0.71,1.15)$ & \\
& More than 3 portions & $0.46(0.27,0.81)$ & \\
\hline
\end{tabular}

*adjusted for dietary factors, maternal age and gestational week of urine sample.

Milk and eggs are important dietary sources of iodine in pregnancy and although seafood was related to iodine status in the unadjusted analysis, the association disappeared after adjustment for other factors. This may be indicative of low seafood consumption in the UK and may possibly reflect the fact that the iodine concentration in a urine sample is related to recent dietary intake (previous 24-48 hours), whereas the FFQ gives a more general overview of dietary intake, so some food items may fail to show associations. Our results should be interpreted with a degree of caution because of the inherent limitations of dietary assessment from food-frequency questionnaires.

This work was partly supported by the European Community's 7th Framework Programme (FP7/2008-2013) grant agreement no. 212652 (NUTRIMENTHE "The Effect of Diet on the Mental Performance of Children").

1. WHO, UNICEF \& ICCIDD (2007) Geneva: World Health Organisation.

2. Bath SC, Steer C, Golding J et al. (2013) Lancet, In press.

3. Golding J, Pembrey M \& Jones R (2001) Paediatr Perinat Epidemiol 15, 74-87.

4. Rogers I \& Emmett P (1998) Eur J Clin Nutr 52, 246-250. 